UQAM-PHE-98/08

UMN-TH-1730/98

TPI-MINN-98/26

December 1998

\title{
The limits on CP-odd four-fermion operators containing strange quark field
}

\author{
C. Hamzaoui ${ }^{a}$ and M. Pospelov ${ }^{b}$ \\ a Département de Physique, Université du Québec à Montréal \\ C.P. 8888, Succ. Centre-Ville, Montréal, Québec, Canada, H3C 3P8 \\ b Theoretical Physics Institute, 431 Tate Laboratory of Physics \\ Minneapolis, MN 55455 USA
}

\begin{abstract}
The bounds on the neutron electric dipole moment and T-odd nucleon-nucleon interaction are used to extract the limits on the effective CP-odd four-fermion operators containing strange quark field. This completes the study of the $\operatorname{dim}=5,6 \mathrm{CP}$-odd operators built from the lightquark fields. The limits are very strong and comparable to those obtained previously for operators containing up and down flavors. We also analyze the shift of the axionic vacuum, $\theta_{\text {eff }}$, induced by four-fermion operators in the presence of $\mathrm{PQ}$ mechanism and conclude that this gives subleading contributions to CP-odd observables as compared with the direct ones.
\end{abstract}

\footnotetext{
${ }^{1}$ hamzaoui@mercure.phy.uqam.ca

${ }^{2}$ pospelov@tpi1.hep.umn.edu
} 


\section{Introduction}

The remarkable accord of standard model (SM) predictions with experiment does not remove the question of a more fundamental theory which would comprise SM as the low energy limit. Among various ways to look for the signs of a new theory, precise tests of fundamental discrete symmetries play an important role.

The nil results for the electric dipole moments of the neutron, heavy atoms and diatomic molecules [1, 2, 3, 4] put in general very strong constraints on the CP-violating sector of a new theory and probe the energy scales unaccessible for direct observations at colliders [5]. Regardless what the particular construction for the new theory is, its relevant contribution at $1 \mathrm{GeV}$ can be reexpressed in terms of effective operators of different dimensions suppressed by corresponding power of a high scale $M$ where these operators were generated:

$$
\mathcal{L}_{e f f}=\sum_{n \geq 4} \frac{c_{n i}}{M^{n-4}} \mathcal{O}_{i}^{(n)}
$$

Here $\mathcal{O}_{i}^{(n)}$ are operators of dimension $n$ and $i$ stands for their different field content, Lorentz structures etc. Fields, relevant for low-energy dynamics, are gluons, three light quark fields, $u, d$ and $s$, and the electromagnetic field. The specifics of a given model enters only through the value of the coefficients $c_{n i}$

$\operatorname{Dim}=3,4$ operators can be combined to form $\theta$-term. In the absence of axion relaxation mechanism this operator is normally the most important on account of possible tree-level contributions $\sim 1$. If $\mathrm{PQ}$ mechanism is operative $\theta \sim 1$ is removed but $\theta$-parameter still can gain a nonzero value induced at low energy by CP-odd operators of bigger dimension. Dim $=5$ operators, which are usually suppressed by an additional $m_{q} / M$ ratio, are electric and chromoelectric dipole moments of quarks. Due to this additional mass ratio, these operators are suppressed by a large scale exactly as $\operatorname{dim}=6$ operators built from four quark fields or purely from gluons (Weinberg operator). Most of the operators have been extensively studied in the literature [6, 7, 5] and limited from experiment using PCAC and QCD sum rules techniques. For the operators with strange quarks, however, only the analysis of the chromoelectric dipole moment is available [9]. Recently some of the four-fermion operators with $s$-quark induced at a high scale in $S U(3) \times S U(2) \times U(1)$-symmetric form were limited using the fact these operators can be mixed with electric dipole moment operators for $u$ and $d$ fields at one-loop level [8]. 
In this letter we combine PCAC approach and the experimental bounds on neutron, mercury and thallium EDMs to put the limits on four-fermionic operators containing strange quark field. This completes the study of the relevant operators $\operatorname{dim}=6$ and can be used for

any model where these operators are generated. Another issue addressed here is the shift of axion vacuum by effective CP-odd four-fermion operators. This shift, $\theta_{\text {eff }}$ is estimated within the same approach .

\section{CP-odd operators containing strange quark}

In what follows, we adopt the classification of operators proposed in Refs. [6, 7, 5]. Among the flavour-conserving CP-odd operators dim $=6$

$$
\begin{aligned}
& \kappa_{1} \frac{G}{\sqrt{2}}(\bar{s} s)\left(\bar{q} i \gamma_{5} q\right) ; \quad \kappa_{2} \frac{G}{\sqrt{2}}\left(\bar{s} i \gamma_{5} s\right)(\bar{q} q) ; \quad \kappa_{3} \frac{G}{\sqrt{2}}\left(\bar{s} i \gamma_{5} s\right)(\bar{s} s) ; \\
& \kappa_{4} \frac{G}{\sqrt{2}}\left(\bar{s} t^{a} s\right)\left(\bar{q} i \gamma_{5} t^{a} q\right) ; \quad \kappa_{5} \frac{G}{\sqrt{2}}\left(\bar{s} i \gamma_{5} t^{a} s\right)\left(\bar{q} t^{a} q\right) ; \quad \kappa_{6} \frac{G}{\sqrt{2}}\left(\bar{s} i \gamma_{5} t^{a} s\right)\left(\bar{s} t^{a} s\right) ; \\
& \kappa_{7} \frac{G}{\sqrt{2}} \frac{1}{2} \epsilon_{\mu \nu \alpha \beta}\left(\bar{s} \sigma_{\mu \nu} s\right)\left(\bar{q} \sigma_{\alpha \beta} q\right) ; \quad \kappa_{8} \frac{G}{\sqrt{2}} \frac{1}{2} \epsilon_{\mu \nu \alpha \beta}\left(\bar{s} \sigma_{\mu \nu} t^{a} s\right)\left(\bar{q} \sigma_{\alpha \beta} t^{a} q\right) ; \\
& \kappa_{9} \frac{G}{\sqrt{2}}(\bar{s} s)\left(\bar{e} i \gamma_{5} e\right) ; \quad \kappa_{10} \frac{G}{\sqrt{2}}\left(\bar{s} i \gamma_{5} s\right)(\bar{e} e) ; \quad \kappa_{11} \frac{G}{\sqrt{2}} \frac{1}{2} \epsilon_{\mu \nu \alpha \beta}\left(\bar{s} \sigma_{\mu \nu} s\right)\left(\bar{e} \sigma_{\alpha \beta} e\right) .
\end{aligned}
$$

we take those, containing $s$-quark field. We will numerate these operators $\mathcal{O}_{i}$ according to the constant $\kappa_{i}$ standing in front of them. $G$ in these formulae is Fermi constant.

As an experimental input we use the following limits, obtained for the neutron EDM [1],

$$
d_{N}<10^{-25} e \cdot c m
$$

and mercury EDM [2] experiments:

$$
d_{H g}<9 \cdot 10^{-25} e \cdot \mathrm{cm}
$$

The latter translates into the limit on the Schiff moment of ${ }^{199} \mathrm{Hg}$ nucleus and eventually leads to the following bound on the effective CP-violating $\pi^{0} \mathrm{pp}$ coupling [2, 5]:

$$
\bar{g}_{\pi p p}<2 \cdot 10^{-11}
$$

To evaluate the contribution of operators (3) to the effective coupling $\bar{g}_{\pi p p}$ we use the same method proposed earlier in Refs. [6, 7, 5]. The operator $\mathcal{O}_{1}$ is the simplest in this respect. 
Using the PCAC reduction of the soft-pion field and calculating subsequent commutators, we reduce the contribution of the $(\bar{s} s)\left(\bar{q} i \gamma_{5} q\right)$ operator $\mathcal{O}_{1}$ to the matrix element of $\bar{s} s \bar{q} q$ operator over the proton:

$$
\left\langle p \pi^{0}\left|\bar{s} s \bar{d} i \gamma_{5} d\right| p\right\rangle=\frac{1}{f_{\pi}}\langle p|\bar{s} s \bar{d} d| p\rangle
$$

This matrix element can be estimated using vacuum insertion approximation:

$$
\langle p|\bar{s} s \bar{d} d| p\rangle \simeq\langle 0|\bar{q} q| 0\rangle\langle p|\bar{s} s+\bar{q} q| p\rangle \simeq 5(1+\beta) \bar{p} p
$$

where we take $\langle p|\bar{d} d| p\rangle \simeq\langle p|\bar{u} u| p\rangle=\langle p|\bar{q} q| p\rangle \simeq 5 \bar{p} p$. The analysis of the barion mass splitting and experimental data on pion-nucleon scattering suggest that the coefficient $\beta, \beta=$ $\langle p|\bar{s} s| p\rangle /\langle p|\bar{q} q| p\rangle$, numerically is close to 0.6 [9, 10]. Thus, CP-violating coupling constant $\bar{g}_{\pi p p}$ is:

$$
\bar{g}_{\pi p p}=5(1+\beta) \frac{\langle 0|\bar{q} q| 0\rangle}{f_{\pi}} G \simeq 8 \cdot 10^{-6} \kappa_{1} .
$$

The rest of the four-quark operators give suppressed contributions to the effective Tviolating nucleon-nucleon interaction. They either contribute only in $\eta$-exchange channel or do not work in vacuum factorization approach. To get the limits on these operators we use the neutron EDM bound. The neutron EDM can be induced as a result of the chiral loop, Fig. 1, where CP-violation resides in one of the meson-nucleon vertecies. In the limit of exact chiral symmetry this loop is logarithmically divergent in the infrared [11] which justifies its appearance in the chiral theory. For our purposes we choose $\Sigma^{-} K^{+}$loop [9] where the operators containing $s$-quark will most likely contribute. In the real life, chiral symmetry is broken and the mass of kaons is rather large, so that estimated limit on $n \Sigma^{-} K^{+}$ coupling has rather large uncertainty:

$$
g_{n \Sigma^{-} K^{+}}<2 \cdot 10^{-11}
$$

Most of the quark operators from the set (3) induce this coupling; to calculate their effect on it we use the same method, PCAC and vacuum factorization. Thus, for example, $\bar{s} i \gamma_{5} s \bar{d} d$ operator contributes in the CP-odd vertex of interest in the following way:

$$
\begin{aligned}
\left\langle\Sigma^{-} K^{+}\left|\bar{s} i \gamma_{5} s \bar{d} d\right| n\right\rangle= & \frac{i}{f_{K}}\left\langle\Sigma^{-}|\bar{d} d \bar{s} u| n\right\rangle \simeq \frac{i}{f_{K}}\langle 0|\bar{q} q| 0\rangle\left\langle\Sigma^{-}|\bar{s} u| n\right\rangle \simeq \\
& \frac{i\langle 0|\bar{q} q| 0\rangle}{f_{K}} \frac{m_{\Sigma}-m_{N}}{m_{s}} \bar{\Sigma}^{-} n \simeq \frac{i\langle 0|\bar{q} q| 0\rangle}{f_{K}} 1.3 \bar{\Sigma}^{-} n
\end{aligned}
$$


and the $S U(3)$-octet type of splitting in the barion mass spectrum was used in the second line of Eq. (10).

The limits on semileptonic operators, $\mathcal{O}_{9}, \mathcal{O}_{10}$ and $\mathcal{O}_{11}$, can be obtained from the limits on T-odd nucleon-electron interaction [5] by simply taking matrix elements from their strangequark part over the nucleon. For the case of $\mathcal{O}_{9}$ and $\mathcal{O}_{10}$ these matrix elements can be easily obtained within the same PCAC approach. In the case of $\mathcal{O}_{11}$, however, the tensor charge of the strange quark over the nucleon, $\left\langle N\left|\bar{s} \sigma_{\mu \nu} s\right| N\right\rangle$, is not known. It is not reducible to the $s$-quark spin content over the nucleon, as it was asserted in Ref. [12], because the latter is expressed by absolutely independent quantity $\left\langle N\left|\bar{s} \gamma_{\mu} \gamma_{5} s\right| N\right\rangle$. Moreover, unlike axialvector operator, tensor operator is odd under charge conjugation and we expect the effects of strange and anti-strange quarks to cancel each other in the first approximation. The model calculations and lattice simulations for tensorial charges give indeed a very suppressed value for the strange quark contribution [13]. The same refers, of course, to the strange quark EDM operator, as it was discussed in [14].

The resulting limits on the coefficients are summarized in Table 1. One can easily see that the best sensitivity is for $\mathcal{O}_{1}$ and $\mathcal{O}_{9}$ operators where $s$-quark enters only as $\bar{s} s$ and does not take part in spin dynamics.

\section{Effective theta-term induced by CP-odd four-fermion operators}

In all known models with significant amount of CP-violation in the flavor-conserving channel, the operators of $\operatorname{dim}>4$ are usually accompanied by a large contribution to the theta term. In other words, $\theta_{\text {loop }}$ is usually by far more sensitive to the new CP-violating physics because it corresponds to the operator $\operatorname{dim}=4$ and therefore need not experience scale suppression of the order $\left(\Lambda_{Q C D} / M\right)^{2}$. Thus the CP-violating operators of $\operatorname{dim}>4$ generated at a scale $M \sim M_{W}$ and higher are important only in the case when $\theta$-term is removed by an axion mechanism. We also assume here the existence of PQ mechanism. In the absence of CP violation, non-removable by PQ transformation, PQ symmetry sets theta parameter to zero [16]. The situation is different in the presence of extra CP-violating sources, communicated by the operators $\operatorname{dim} \geq 5$. These operators $\mathcal{O}_{i}$ shift the axion vacuum and generate additional indirect contribution to all CP-odd observables through the effective $\theta$-term given by the ratio 
of two correlators:

$$
\begin{aligned}
\theta_{\text {eff }} & =-\frac{K_{i}}{|K|}, \text { where } K=i\left\{\int d x e^{i k x}\left\langle 0\left|T\left(\frac{\alpha_{s}}{8 \pi} G \tilde{G}(x), \frac{\alpha_{s}}{8 \pi} G \tilde{G}(0)\right)\right| 0\right\rangle\right\}_{k=0} \\
K_{i} & =i\left\{\int d x e^{i k x}\left\langle 0\left|T\left(\frac{\alpha_{s}}{8 \pi} G \tilde{G}(x), \mathcal{O}_{i}(0)\right)\right| 0\right\rangle\right\}_{k=0} .
\end{aligned}
$$

Here $G_{\mu \nu}^{a} \tilde{G}_{\mu \nu}^{a}$ is abbreviated as $G \tilde{G}$. The calculation of $K$ is based on the use of the anomaly equation and the saturation of subsequent correlators by light hadronic states [15]:

$$
K=\frac{m_{\pi}^{2} f_{\pi}^{2} m_{u} m_{d}}{2\left(m_{u}+m_{d}\right)^{2}} .
$$

( $F_{\pi}$ we use throughout the paper is $130 \mathrm{MeV}$ ). The same technique can be exploited in the case of $K_{1}$. For the case of chromoelectric dipole moments the explicit derivation of $K_{i}$ can be found in Ref. [17, 18]. A similar type of calculation could be done for the most part of the four-fermion operators discussed here and in earlier works [6, 7]. Using the anomaly equation in the form

$$
\begin{array}{r}
\partial_{\mu} \frac{m_{d} m_{s} \bar{u} \gamma_{\mu} \gamma_{5} u+m_{u} m_{s} \bar{d} \gamma_{\mu} \gamma_{5} d+m_{u} m_{d} \bar{s} \gamma_{\mu} \gamma_{5} s}{m_{s} m_{d}+m_{s} m_{u}+m_{d} m_{u}}= \\
\frac{2 m_{u} m_{d} m_{s}}{m_{s} m_{d}+m_{s} m_{u}+m_{d} m_{u}}\left(\bar{u} \gamma_{5} u+\bar{d} \gamma_{5} d+\bar{s} \gamma_{5} s\right)+\frac{\alpha_{s}}{4 \pi} G \tilde{G},
\end{array}
$$

we apply standard technique of current algebra. The correlators of interest, $K_{i}$, can be rewritten in the form of the equal-time commutator, which for all sets of four-fermion operators we can calculate easily, plus the term containing the singlet combination of pseudoscalars built from quark fields. Thus, for $\mathcal{O}_{1}$ operator we have the following expression:

$$
\begin{gathered}
K_{1}=\kappa_{1} \frac{G}{\sqrt{2}}\left\langle 0\left|\frac{m_{d} m_{s}(\bar{u} u)(\bar{s} s)}{m_{s} m_{d}+m_{s} m_{u}+m_{d} m_{u}}+\frac{m_{u} m_{d}\left(\bar{u} i \gamma_{5} u\right)\left(\bar{s} i \gamma_{5} s\right)}{m_{s} m_{d}+m_{s} m_{u}+m_{d} m_{u}}\right| 0\right\rangle+ \\
\int d^{4} x\left\langle 0\left|T\left\{\frac{i m_{u} m_{d} m_{s}}{m_{s} m_{d}+m_{s} m_{u}+m_{d} m_{u}}\left(\bar{u} \gamma_{5} u+\bar{d} \gamma_{5} d+\bar{s} \gamma_{5} s\right)(x), \mathcal{O}_{1}(0)\right\}\right| 0\right\rangle
\end{gathered}
$$

The second line here is suppressed by an extra power of light quark masses in the numerator. It would bring a comparable contribution, though, if there were an intermediate hadronic state with a mass, vanishing in the chiral limit $m_{i} \rightarrow 0$. At the same time, the flavor structure of this term shows that the lightest intermediate state here is $\eta^{\prime}$ which is believed to remain heavy even if quark masses vanish. Thus the contribution from the second term is negligible in the limit $m_{\pi} \ll m_{\eta^{\prime}}$. The second term in the first line of Eq. (15) is suppressed 
by $m_{u} / m_{s}$ ratio and effectively we get the following formula for the induced theta term due to the operator $\mathcal{O}_{1}$ :

$$
\theta_{\text {eff }}=-\kappa_{1} \delta_{1} \frac{G}{\sqrt{2}} m_{u}^{-1}\langle 0|\bar{q} q| 0\rangle,
$$

where $\delta_{1}$ is the ratio of the four-quark condensate to the square of $\bar{q} q$ condensate.

$$
\delta_{1}=\frac{\langle 0|\bar{u} u \bar{s} s| 0\rangle}{\langle 0|\bar{q} q| 0\rangle^{2}} .
$$

In the case of $\mathcal{O}_{1}$ we can use vacuum factorization and estimate that $\delta_{1} \sim 1$. For some of four-quark operators vacuum factorization does not work and we expect $\delta_{i}$ to be smaller than 1 . The appearence of $m_{u}^{-1}$ in Eq. (15) is because the operator $\mathcal{O}_{1}$ breaks chirality. Any answer for CP-odd observables, iduced by $\theta$ will not contain this singularity.

The value of $\theta_{\text {eff }}$ induced by $\mathcal{O}_{1}$ leads to an additional contribution to $\bar{g}_{\pi p p}$ constant,

$$
\bar{g}_{\pi p p}\left(\theta_{e f f}\right)=\frac{m_{u} m_{d}}{m_{u}+m_{d}} \frac{\sqrt{2} \theta_{e f f}}{f_{\pi}}=\delta_{1} \kappa_{1} \frac{G\langle 0|\bar{q} q| 0\rangle}{f_{\pi}} \frac{2 m_{d}}{m_{u}+m_{d}}=1.3 \cdot 10^{-6} \kappa_{1},
$$

which should be compared with the direct contribution (8). We see that in the case of $\mathcal{O}_{1}$ the indirect contribution related to theta term gives $15-20 \%$ correction to the CP-odd vertex $\bar{g}_{\pi p p}$. In fact, this is the biggest value of $\theta_{\text {eff }}$, generated by the set of operators (3). This is especially true for the operators $\mathcal{O}_{3}$ and $\mathcal{O}_{6}$ composed exclusively from strange quark which induce $\theta_{\text {eff }}$ with an additional parametrical suppression $\left(m_{u}^{-1}+m_{d}^{-1}\right) / m_{s}$. We have also done similar calculation for the operators composed from the $u$ and $d$ field [6, 7, 5] and the result for the $\theta$-driven contributions never exceeds $20 \%$ from the direct contribution. The estimates for $\theta_{\text {eff }}$ are included into Table 1.

\section{Conclusions}

We have considered the limits on the four-fermion CP-odd operators containing strange quarks coming from the neutron, thallium and mercury EDM experiments. This completes the study of CP-odd dim=5,6 operators built from light-quark fields. We observe that the limits are very strong, especially for the operators $\bar{s} s \bar{q} i \gamma_{5} q$ and $\bar{s} s \bar{e} i \gamma_{5} e$ where strange quark is in some sense "spectator". The limits summarized in Table 1 are almost as strong as for operators composed from $u$ and $d$ quarks. This is because the strange quark condensate is the same as for up and down quarks in the flavor $S U(3)$ symmetry approximation and the

content of the $s$-quark in the nucleon in scalar, pseudoscalar and axial-vector channels is also 
significant. The limits on the operators $\mathcal{O}_{1}, \mathcal{O}_{9}$ and $\mathcal{O}_{10}$ are extracted with much smaller error than those for the rest of the operators. Other limits are estimated within an order of magnitude, mainly because of the chiral loop, used to obtain the EDM of the neutron. The infrared logarithm enhancement factor, $\log m_{K}$, is numerically important only in the limit $m_{K} \rightarrow 0$.

There is an alternative method of limiting four-fermion operators used in Ref. [8]. In this work different linear combination of operators (3) were taken to form a different set, invariant under standard model group. At one-loop level some of these operators can be mixed with EDMs of $u$ and $d$ quarks with the coefficients proportional to $m_{s} \log (\Lambda / 1 \mathrm{GeV})$. The comparison of the limits obtained in Ref. [8] and in the present work shows that they are complementary. For most of the operators the limits obtained here are stronger, although $\mathcal{O}_{\text {, }}$ and especially $\mathcal{O}_{\infty \infty}$ can be better constrained from their one-loop mixing with quark and electron EDM operators.

The shift of the axionic vacuum induced by four-fermion operators is shown to give contributions to CP-odd observables normally smaller than the direct contributions not

associated with $\theta_{\text {eff }}$. For some operators this type of correction can be as large as 15-20\% from the direct contribution.

\section{Acknowledgments}

M.P. would like to thank P. Herczeg and I. B. Khriplovich for valuable discussions. This work is supported in part by N.S.E.R.C. of Canada and DOE grant DE-FG02-94ER-40823 at the University of Minnesota.

\section{References}

[1] K.F. Smith et al., Phys. Lett. B234 (1990) 191; I.S. Altarev et al., Phys. Lett. B276 (1992) 242.

[2] J.P. Jacobs et al., Phys. Rev. Lett. 71 (1993) 3782.

[3] E.D. Commins et al., Phys. Rev. A50 (1994) 2960.

[4] D. Cho, K. Sangster and E. Hinds, Phys. Rev. Lett., 63 (1989) 2559. 
[5] I.B. Khriplovich and S.K. Lamoreaux, "CP Violation Without Strangeness", Springer, 1997.

[6] V.M. Khatsimovsky, I.B. Khriplovich and A.S. Yelkhovsky, Ann. Phys. 186 (1988) 1.

[7] V.M. Khatsimovsky and I.B. Khriplovich, Phys. Lett. B296 (1994) 219.

[8] X.-G. He and B. McKellar, Phys. Lett. B390 (1997) 318.

[9] V.M. Khatsimovsky, I.B. Khriplovich and A.R. Zhitnitsky, Z. Phys. C36 (1987) 455.

[10] A. Zhitnitsky, Phys. Rev. D55 (1997) 3006.

[11] R. Crewther et al., Phys. Lett. B88 (1979) 123.

[12] J. Ellis and R. Flores, Phys. Lett. B377 (1996) 83.

[13] H.-C. Kim, M.V. Polyakov and K. Goeke, Phys. Lett. B387 (1996) 577; S. Aoki et al., Phys. Rev. D56 (1997) 433.

[14] C. Hamzaoui, M. Pospelov and R. Roiban, Phys. Rev. D56 (1997) 4295.

[15] M.A. Shifman, A.I. Vainshtein and V.I. Zakharov, Nucl. Phys. B166 (1980) 493.

[16] R.D. Peccei and H. Quinn, Phys. Rev. Lett. 38 (1977) 1440.

[17] I. Bigi and N.G. Uraltsev, Sov. Phys. JETP 100 (1991) 198.

[18] M. Pospelov, Phys. Rev. D58 (1998), 097703.

[19] W.A. Bardeen and S.H.H. Tye, Phys. Lett. B74 (1978) 580. 
Table 1.

The limits obtained on the $C P$-odd four-fermion operators from neutron, thallium and mercury EDM experiments.

\begin{tabular}{|c|c|c|c|c|c|c|}
\hline & $\mathcal{O}_{1}$ & $\mathcal{O}_{2}$ & $\mathcal{O}_{3}$ & $\mathcal{O}_{4}$ & $\mathcal{O}_{5}$ & $\mathcal{O}_{6}$ \\
\hline$\kappa_{i}$ & $3 \cdot 10^{-6}$ & $7 \cdot 10^{-5}$ & $7 \cdot 10^{-5}$ & $3 \cdot 10^{-4}$ & $3 \cdot 10^{-4}$ & $3 \cdot 10^{-4}$ \\
\hline$\left|\theta_{\text {eff }}\right|$ & $5 \cdot 10^{-5} \kappa_{1}$ & $5 \cdot 10^{-5} \delta_{2} \kappa_{2}$ & $2 \cdot 10^{-6} \kappa_{3}$ & $5 \cdot 10^{-5} \delta_{4} \kappa_{4}$ & $5 \cdot 10^{-5} \delta_{5} \kappa_{5}$ & $5 \cdot 10^{-7} \kappa_{6}$ \\
\hline
\end{tabular}

\begin{tabular}{|c|c|c|c|c|c|}
\hline & $\mathcal{O}_{7}$ & $\mathcal{O}_{8}$ & $\mathcal{O}_{9}$ & $\mathcal{O}_{10}$ & $\mathcal{O}_{11}$ \\
\hline$\kappa_{i}$ & $3 \cdot 10^{-5}$ & $2 \cdot 10^{-5}$ & $2 \cdot 10^{-7}$ & $10^{-5}$ & $10^{-5}$ \\
\hline$\left|\theta_{\text {eff }}\right|$ & $5 \cdot 10^{-5} \delta_{7} \kappa_{7}$ & $5 \cdot 10^{-5} \delta_{8} \kappa_{8}$ & - & - & - \\
\hline
\end{tabular}




\section{Figure captions.}

Chiral loop diagrams, inducing the EDM of the neutron. Dirac structure of $C P$-violating vertex is proportional to 1 . This diagram logarithmically diverges in the limit $m_{K} \rightarrow 0$.
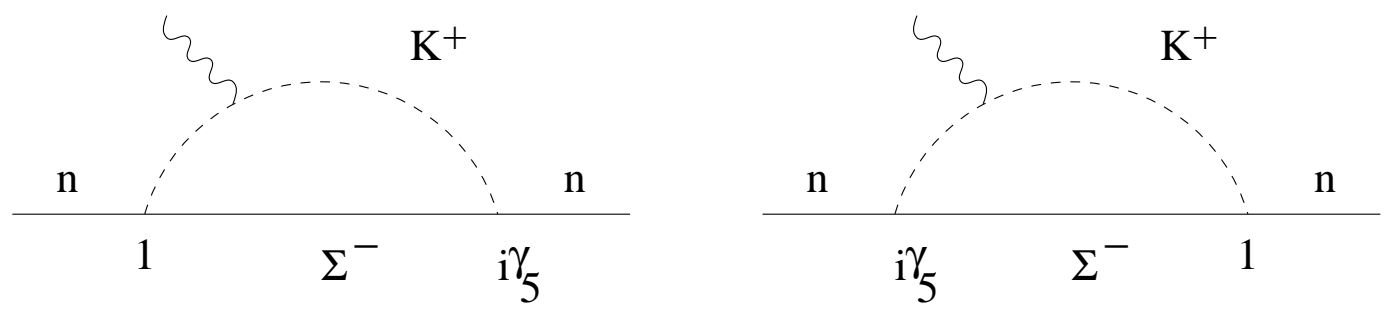

Fig. 1 